\section{CORPUS PUBLISHERS}

\section{Current Research} in Emergency Medicine (CREM)

Volume 2, Issue 2, 2022

Article Information

Received date : 07 February, 2022

Published date: 04 March, 2022

\section{*Corresponding author}

Alawi Al-Mashhor A, MBBS, MRCP, MRCEM, Emergency Medicine Registrar, Royal Albert Edward Infirmary Hospital, Wigan, Greater Manchester, UK

\section{Key Words}

Emergency Department; Mortality;

Pneumonia; Transthoracic

Echocardiography; Flank Pain;

Contralateral Pathology; Transthoracic

Echocardiography

Distributed under: Creative Commons CC-BY 4.0

\title{
Left Flank Pain: is it Renal Colic Or Contralateral Pathology...?
}

\author{
Alawi Al-Mashhor ${ }^{1 \oplus}$, Abdulhadi Tashkandi ${ }^{\bullet}$, Mohammed Anjum Ahmed $^{3 \oplus}$ \\ Murtaza Anjum Ahmed ${ }^{4 \oplus}$, Ahmed Mnofala ${ }^{\mathfrak{\varpi}^{\oplus}}$ \\ ${ }^{I} \mathrm{MBBS}, \mathrm{MRCP}, \mathrm{MRCEM}$, Emergency Medicine Registrar, Royal Albert Edward Infirmary Hospital, Wigan, \\ Greater Manchester, UK \\ ${ }^{2}$ Consultant \& Chairman of Emergency Department, Prince Mohammed Bin Abdulaziz Hospital,National \\ Guard Health Affairs, Al-Madinah Al-Munawwarah, Saudi Arabia \\ ${ }^{3} \mathrm{MBBS}, \mathrm{MRCEM}$, Emergency medicine assistant consultant, rince Mohammed Bin Abdulaziz \\ Hospital,National Guard Health Affairs, Al-Madinah Al-Munawwarah, Saudi Arabia \\ ${ }^{4}$ MBBS, EBEEM, Emergency Medicine Staff Physician, rince Mohammed Bin Abdulaziz Hospital,National \\ Guard Health Affairs, Al-Madinah Al-Munawwarah, Saudi Arabia \\ ${ }^{5}$ MBBS, Emergency Medicine Staff Physician, rince Mohammed Bin Abdulaziz Hospital,National Guard \\ Health Affairs, Al-Madinah Al-Munawwarah, Saudi Arabia
}

\section{Abstract}

\section{Background}

Acute aortic dissection is an uncommon but life-threatening emergency, which is often missed in up to $38 \%$ of patients on initial evaluation, and in up to $28 \%$ of patients, the diagnosis is made at autopsy. Painless aortic dissection has been reported, but is relatively uncommon. The mortality rates are estimated at $50 \%$ by 48 hours and increase by $1 \%$ per hour if undiagnosed.

\section{Case presentation}

We report a case of atypical aortic dissection who presented to ER with subtle unspecific contralateral renal colicky like pain as a primary symptom, which had made the prompt diagnosis very challenging and difficult. Patient had no history of any chronic disease. Vital signs and abdominal examination were normal. Provisional diagnosis of renal colic was made, analgesic was given to control the pain. Laboratory result showed (4+) RBCS in point of care strip testing otherwise were unremarkable, and CT KUB was requested. Provisional CT-KUB reported by radiologist as unremarkable for any genitourinary pathology. On careful review of the above plain CT by emergency physician, there was ecstatic flap like calcification of thoraco-abdominal aorta. Urgent CT with contrast was requested which confirmed Stanford B aortic dissection.

\section{Conclusion}

Proper diagnosis of acute aortic dissection can be difficult when patients present atypically, especially with subtle unspecific symptoms. Aortic dissection is to be considered, even without the presence of characteristic pain in elderly specifically and high-risk patients. A heightened level of attention with low threshold of requesting proper imaging elderly or those with high-risk patient with back or flank pain, are needed for better diagnosis and exclude other catastrophic causes like aortic dissection. Also emphasizing on emergency physicians review of the images in emergency department and integration of radiology training in emergency medicine program, which helps in prompt diagnosis.

\section{Introduction}

Flank pain is a common complaint in the emergency department with a variety of causes. The causes could be more severe such as renal calculus and extend to critical such as abdominal aortic aneurysm or aortic dissection. Overall, acute ureteral obstructions are the most common and disturbing cause. Due to the complex innervation pattern of the flank, pain can arise from a number of different organ systems [1]. Multiple studies have shown that after using non-contrast Computed Tomography (CT) scans in patients who were thought to have renal colic, $\sim 10 \%$ are ultimately given an alternate diagnosis [2]. Acute aortic dissection is a relatively rare but life-threatening medical emergency, and can be extremely difficult to diagnose, especially with atypical presentation. The mortality rates are estimated at $20 \%$ by 48 hours and increase by $1 \%$ per hour if undiagnosed [3]. The outcome is usually fatal with rapid development of serious complications. Clinical manifestations of acute aortic dissection are diverse in the general population. According to the previous report only $72.7 \%$ of patients present with typical textbook presentations such as severe tearing chest pain [4]. There is still a significant percentage of patients presenting with diverse atypical signs and symptoms, which might mislead the first line physician to misdiagnose such a lethal condition.

Case Report

A 63 years old male patient presented to our health care facility with history of left sided flank pain, colicky in nature radiating down to the center abdomen and left iliac fossa for two days, sudden in onset. No urinary symptoms, only reported constipation then. Vital signs were normal. Patient has no history of any chronic disease. Provisional diagnosis of renal colic was made, analgesic were given to control pain and investigation requested accordingly. Pain improved after analgesia. Laboratory result showed (4+) RBCS in point of care strip testing otherwise were unremarkable, and CT KUB was requested. Following to the CT-KUB, patient report pain increased. On examination he was noted to have pain score of 7 and elevated systolic blood pressure of $170 / 95 \mathrm{mmHg}$. Provisional CT-KUB reported by radiologist as unremarkable for any genitourinary pathology. On careful review of the above plain CT by emergency physician, there was ecstatic flap like calcification of thoraco-abdominal aorta, which were seen in several cuts in CT KUB (figures 1 and 2). Bedside ultrasound performed by the emergency physician, which showed suspicion of moving flap in abdominal aorta. Urgent CT with contrast was 
requested which revealed typical appearance of Tennis ball sign(figure 3), parrot beak sign(figure 4) and confirming Stanford B aortic dissection, starting from proximal aortic arch passing through descending thoracic aorta, abdominal aorta(figures 5-8), proximal right renal artery and right common iliac and external iliac artery Management of aortic dissections including controlling blood pressure were initiated and urgent vascular surgery team consultation was requested.

Radiological finding

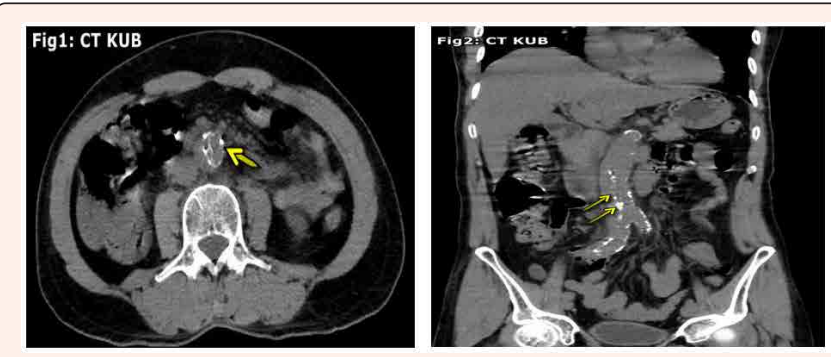

Figure 1 \& 2: CT KUB showed ecstatic flap like calcification of thoracoabdominal aorta. (Yellow arrows)

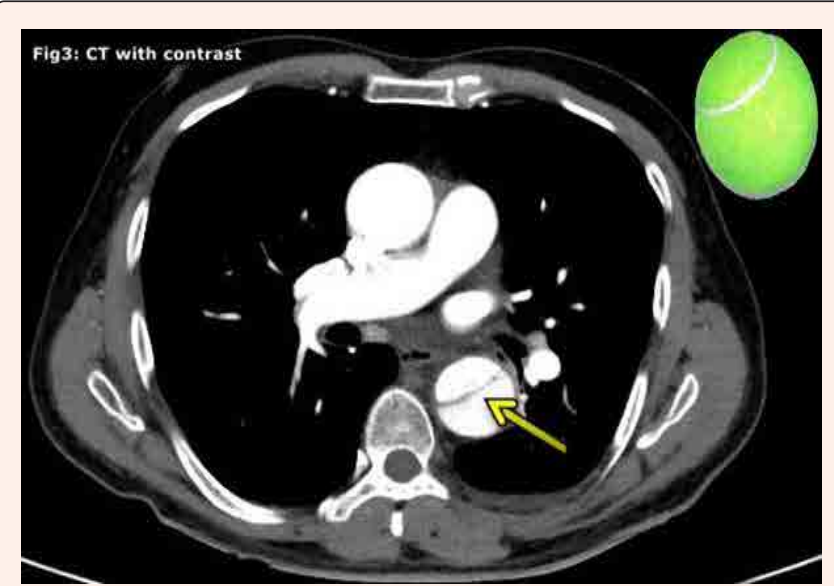

Figure 3: Tennis ball appearance

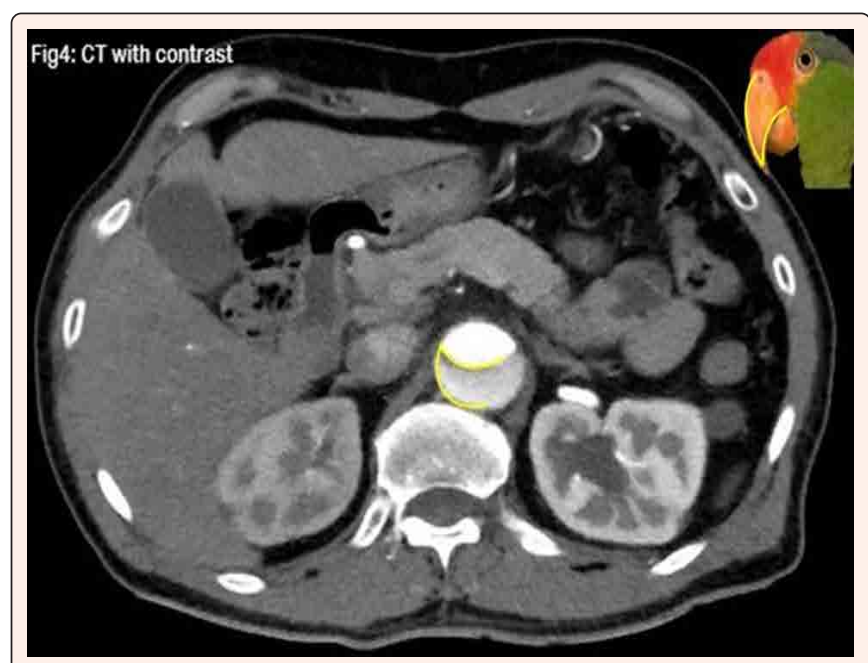

Figure 4: Parrot beak appearance
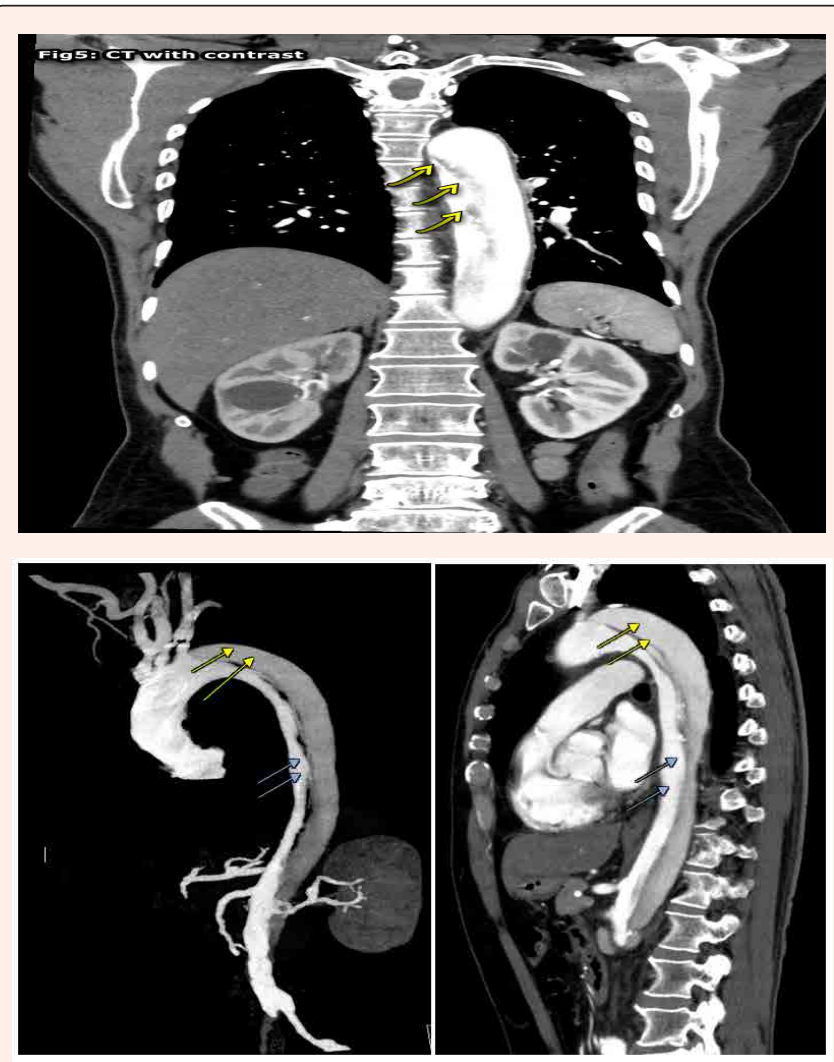

Fig 5-6-7(CT contrast): Thoracic dissection (yellow arrows) with abdominal extension (blue arrow)

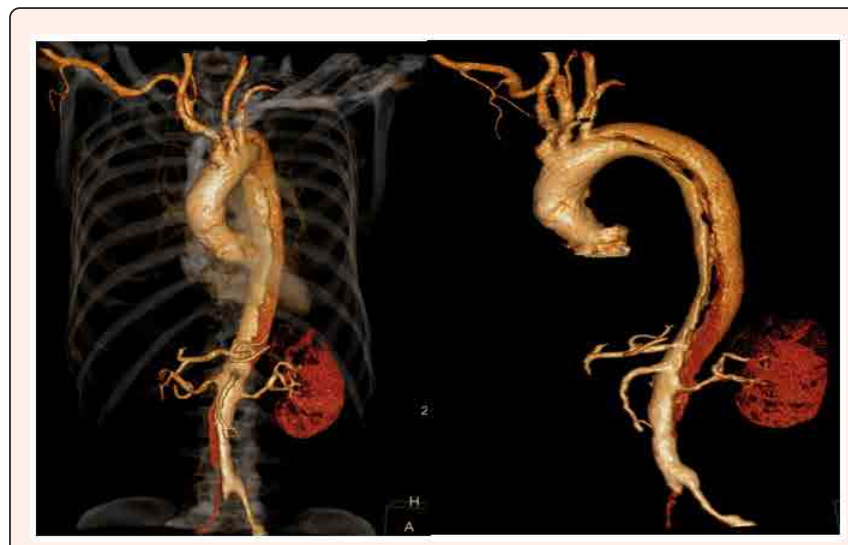

Figure 8: 3D image of Dissection

\section{Discussion}

Acute aortic dissection is missed in up to $38 \%$ of patients on initial evaluation, and in up to $28 \%$ of patients the diagnosis is made at autopsy [5]. A 2011 report estimates an incidence of acute aortic dissection is three or four cases per 100,000 people per year [6]. According to the International Registry of Acute Aortic Dissection (IRAD), the typical patient with acute aortic dissection is a male in his 70 s with a history of hypertension, who presents with an abrupt onset of tearing, cutting, or shearing chest pain. Several case series found that only $\sim 60-70 \%$ of patients present with typical manifestations. About $6 \%$ of patients with painless AAD had syncope, congestive heart failure, or stroke and have higher mortality [7]. The clinical manifestations of acute aortic dissection are diverse in the general population. Different symptoms occur because of perfusion defect of the brain, limbs, and visceral organs. Thus, there 
Table 1

\begin{tabular}{|c|c|c|c|c|c|}
\hline \multirow{2}{*}{ Non trauma CTs } & \multicolumn{5}{|c|}{ Emergency medicine physician-attending radiologists of non-trauma CT } \\
\hline & Sensitivity \% & Specificity \% & Positive predictive value & Negative predictive value & Area under curve \\
\hline \multicolumn{6}{|l|}{ Cranial CT $(\mathrm{n}=92)$} \\
\hline Brain hemorrhage $(\mathrm{n}=39)$ & $92.31-97.44$ & $100-100$ & $100-100$ & $94.6-98.1$ & $0.962-0.987$ \\
\hline \multicolumn{6}{|l|}{ Chest CT $(n=67) /$ angiography $(n=95)$} \\
\hline Spontaneous pneumothorax $(n=6)$ & $100-100$ & $100-100$ & $100-100$ & $100-100$ & $1.00-1.00$ \\
\hline Pulmonary embolism ( $\mathrm{n}=27)$ & $74.1-92.6$ & $95.6-97.1$ & $87.0-92.6$ & $90.3-97.1$ & $0.848-0.948$ \\
\hline Other pathologic finding $(=35)^{*}$ & $91.43-97.14$ & $96.87-100$ & $97-100$ & $91.2-97$ & $0.942-0.986$ \\
\hline \multicolumn{6}{|l|}{ Abdomenal CT $(n=258)$} \\
\hline Kidney and Urinary tract Stones $(\mathrm{n}=23)$ & 73.91-91.30 & $95.08-98.36$ & $85-95.5$ & $90.6-96.8$ & $0.845-0.948$ \\
\hline Acute pancreatitis $(n=9)$ & $55.6-77.80$ & $98.4-100$ & $83.3-100$ & $94-97$ & $0.770-0.889$ \\
\hline Cholecystitis//Cholelithiasis (n=19) & $73.68-89.47 \%$ & $98.31-98.31$ & $93.3-94.4$ & $92.1-96.7$ & $0.860-0.939$ \\
\hline Other pathologic findings $(n=19) * *$ & $57.90-84.20 \%$ & $95.56-100$ & $84.6-100$ & $84.3-93.7$ & $0.767-0.921$ \\
\hline \multicolumn{6}{|l|}{$\begin{array}{l}\text { Contrast-enhanced thoracoabdominal CT } \\
(\mathrm{n}=58)\end{array}$} \\
\hline Aortic aneurysm/ Aortic dissection $(\mathrm{n}=7)$ & $100-100$ & $100-100$ & $100-100$ & $100-100$ & $1.000-1.000$ \\
\hline
\end{tabular}

*Thoracic mass, pneumonia, empyema, pleuraleffusion, lung abscess.

${ }^{*}$ Abdominalmass, abscess, interstitialfluid, pneumoperitoneum, bowel obstruction, diverticuluitis, gynecological disease.

$\mathrm{CT}=$ computed tomography; $\mathrm{PPV}=$ positive predictive value; $\mathrm{NPV}=$ Negative predictive value; $\mathrm{AUC}=\mathrm{Area}$ under curve.

appeared to be a wide range of clinical presentations, which prolongs investigation in the ED and increases the risk of in-hospital death. In fact, it suggests the need for first emergency physicians to be highly attentive when making diagnosis of this lifethreatening disease. In our case, the patient presented with some subtle unspecific symptoms such as left flank pain and left groin pain with hematuria, which had made the diagnosis very difficult and challenging for the emergency physician. Flank pain that mimic ureteral colic pain has no explanation although the dissection is on the contralateral side involving right renal artery and not in left one. The majority of patients with acute aortic dissection had abnormalities in thoracic X-ray. However, 10 to $20 \%$ of the patients had a normal thoracic X-ray, so a normal thoracic X-ray canno rule out the diagnosis. For definitive diagnosis of acute aortic dissection, imaging such as cardiac CT, magnetic resonance imaging (MRI), Trans-Esophageal Echography (TEE) or aortography must be performed. CT is the most often used modality to diagnose of aortic dissection because of its high specificity and sensitivity and it availability. MRI also has high specificity and sensitivity, but the test is time consuming and less available. TEE is usually used for hemodynamically unstable patients since it can be performed at the bedside. In spite of previously reported moderate specificity and sensitivity of Transthoracic Echocardiography (TTE) [8]. The TTE have been described as a useful imaging modality for the diagnosis of classic acute type-A aortic dissection [9]. In order to establish definitive diagnosis, better characterize the aortic dissection and to decide the therapeutic option; CT need to be performed.

According to Aortic Dissection Detection Risk Score developed by American Heart Association, patients with risk score 2 or 3 (abrupt onset back pain, systolic BP differential, murmur of aortic insufficiency) recommended to undergone immediate aortic imaging evaluation and surgical consultation [10].

Values before the hyphen indicate the value of emergency physcicians, where as value after the sign indicate the ratio of attending radiologists.

Accuracy of CT interpretation by emergency physicians reported variable sensitivity and specificity. One study reported CT interpretation sensitivity and specificity levels by emergency physicians above $90 \%$ in patients with non-traumatic injuries and excluding pulmonary embolism. The CT interpretation sensitivity ratios in abdominal injuries vary between $60 \%$ and $80 \%$ with specificity ratios above $95 \%$. The CT interpretation sensitivity ratio of EP for pulmonary embolism was $74.1 \%$ and the specificity ratio was $95.6 \%$ [ above Table 1]. The interpretations of EP regarding CT scans due to nontraumatic pathologies were concluded that the agreement between the final result and brain hemorrhage, spontaneous pneumothorax, thoracic other pathologic findings, cholecystitis/thickened gallbladder wall/cholelithiasis and aortic aneurysm/aortic dissection diagnoses were perfect $(\kappa \geq 0.75)(\kappa=0.933 ; \kappa=1.000$ $\kappa=0.881 ; \kappa=0.775 ; \kappa=1.000$; respectively) [11]. The initial management of an aortic dissection is the same independent of the type. Pain to be controlled as well intravenous agents to be considered to control blood pressure. Definitive management (operative versus conservative) depends on type of dissection. Our patient had a Stanford type $\mathrm{B}$ aortic dissection, patient was managed conservatively with close follow up with vascular surgery services. Overall, in-hospital mortality rates in patients with type B aortic dissection remains high with nearly $26 \%$ of patients with $16 \%$ in aged 70 years or more [12].

\section{Conclusion}

Proper diagnosis of acute aortic dissection can be difficult when patients present atypically, especially with subtle unspecific symptoms. In this case, the patient presented with the signs and symptoms of flank pain on the contralateral site without other obvious causes; aortic dissection to be considered, even without the presence of characteristic pain in elderly specifically and high-risk patients. A heightened level of attention with low threshold of requesting proper imaging such as CT mainly in elderly or those with high-risk patient with back or flank pain, are needed for better diagnosis and exclude other catastrophic causes like aortic dissection. Also emphasizing on emergency physicians review of the images in emergency department and integration of radiology training in emergency medicine program, which helps in prompt diagnosis as evident from the above case. 


\section{References}

1. Smith RC, Levine J, Dalrymple NC, Barish M, Rosenfield AT (1999) Acute flank pain: A modern approach to diagnosis and management, Seminars in Ultrasound, CT and MRI 20(2): 108-135.

2. Katz DS, Scheer M, Lumerman JH, Mellinger BC, Stillman CA, et al. (2000) Alternative or additional diagnoses on unenhanced helical computed tomography for suspected renal colic: experience with 1000 consecutive examinations. Urology 56(1): 53-57.

3. Schulman PM, Lane R (2007) Aortic Dissection. In: Parsons PE, WienerKronish JP (Eds), 2rth (edn.) Critical Care Secrets, Mosby, US, pp. 184-190.

4. Hagan PG, Niebnabar CA, Isselbacher EM, Bruckman D, Karavite J, et al. (2000) The international registry of acute aortic dissection (IRAD) - new insight into an old disease. JAMA 283(7): 897-903.

5. Ayrik C, Cece H, Aslan O, Karcioglu O, Yilmaz E (2006) Seeing the invisible: painless aortic dissection in the emergency setting. Emerg Med J 23(3): e24.

6. LeMaire S, Russell L (2011) Epidemiology of thoracic aortic dissection. Nat Rev Cardiol 8(2): 103-113.

7. Park SW, Hutchison S, Mehta RH, Isselbacher EM, Cooper JV, et al. (2004) Association of Painless Acute Aortic Dissection With Increased Mortality.
Mayo Clin Proc 79(10): 1252-1257.

8. Erbel R, Aboyans V, Boileau C, Bossone E, Bartolomeo RD, et al. (2014) ESC Guidelines on the diagnosis and treatment of aortic diseases: Document covering acute and chronic aortic diseases of the thoracic and abdominal aorta of the adult. The Task Force for the Diagnosis and Treatment of Aortic Diseases of the European Society of Cardiology (ESC). Eur Heart J 35(41): 2873-2926.

9. Cecconi M, Chirillo F, Costantini C, Iacobone G, Lopez E, et al. (2012) The role of transthoracic echocardiography in the diagnosis and management of acute type A aortic syndrome. Am Heart J 163: 112-118.

10. Rogers AM, Hermann LK, Booher AM, Nienaber CA, William DM, et al. (2011) Sensitivity of the aortic dissection detection risk score, a novel guideline-based tool for identification of acute aortic dissection at initial presentation: results from the international registry of acute aortic dissection. Circulation 123(20): 2213-2218.

11. Guven R, Akca AH, Caltili C, Sasmaz MI, Kaykisiz EK, et al. (2018) Comparing the interpretation of emergency department computed tomography between emergency physicians and attending radiologists: A multicenter study. Niger J Clin Pract 21(10): 1323-1329.

12. Mehta RH, Bossone E, Evangelista A, O'Gara PT, Smith DE, et al. (2004) Acute type $\mathrm{B}$ aortic dissection in elderly patients: clinical features, outcomes, and simple risk stratification rule. Ann Thorac Surg 77(5): 1622-1628. 